\title{
New Data and Tools for Integrating Discrete and Continuous Population Modeling Strategies
}

\author{
JAMES S. KOOPMAN, ${ }^{a}$ GEOFFREY JACQUEZ, ${ }^{b}$ AND STEPHEN E. CHICK \\ ${ }^{a}$ Department of Epidemiology and Center for the Study of Complex Systems, \\ University of Michigan, Ann Arbor, Michigan, USA \\ ${ }^{b}$ Biomedware Incorporated, Ann Arbor, Michigan, USA \\ ${ }^{c}$ Department of Industrial and Operations Engineering and Center for the \\ Study of Complex Systems, University of Michigan, Ann Arbor, Michigan, USA
}

\begin{abstract}
Realistic population models have interactions between individuals. Such interactions cause populations to behave as systems with nonlinear dynamics. Much population data analysis is done using linear models assuming no interactions between individuals. Such analyses miss strong influences on population behavior and can lead to serious errors-especially for infectious diseases. To promote more effective population system analyses, we present a flexible and intuitive modeling framework for infection transmission systems. This framework will help population scientists gain insight into population dynamics, develop theory about population processes, better analyze and interpret population data, design more powerful and informative studies, and better inform policy decisions. Our framework uses a hierarchy of infection transmission system models. Four levels are presented here: deterministic compartmental models using ordinary differential equations (DE); stochastic compartmental (SC) models that relax assumptions about population size and include stochastic effects; individual event history models (IEH) that relax the SC compartmental structure assumptions by allowing each individual to be unique. IEH models also track each individual's history, and thus, allow the simulation of field studies. Finally, dynamic network (DNW) models relax the assumption of the previous models that contacts between individuals are instantaneous events that do not affect subsequent contacts. Eventually it should be possible to transit between these model forms at the click of a mouse. An example is presented dealing with Cryptosporidium. It illustrates how transiting model forms helps assess water contamination effects, evaluate control options, and design studies of infection transmission systems using nucleotide sequences of infectious agents.
\end{abstract}

KEYWORDS: new data; new tools; discrete models; continuous models; population modeling

Address for correspondence: James S. Koopman, M.D., Ph.D., University of Michigan, Department of Epidemiology, SPH-1, 109 Observatory St., Ann Arbor, MI 48109, USA. Voice: 734-763-5269; fax: 734-764-3192.

jkoopman@umich.edu 


\section{INTRODUCTION}

Dynamic population models underpin the theoretical structure of population sciences such as epidemiology and demography. Yet many population scientists are not adept at constructing and analyzing such models. We propose here a framework that simplifies complex model construction and facilitates more complete population system analyses.

After presenting an overview, we motivate the need for the set of model forms we propose by discussing five purposes of population system modeling. We present four hierarchically constructed model forms and discuss the use of those four model forms to analyze Cryptosporidia transmission systems in pursuit of the five modeling goals. This includes a discussion of how to use data on nucleotide sequences of infectious agents gathered from individuals with known contact points in a transmission system. Our summary section discusses some implications for advancing population sciences.

\section{OVERVIEW}

Data analysis models used by epidemiologists and demographers typically assume that individuals do not interact. This assumption implies that population dynamics are linear at the population level and, thus, what happens to individuals is dependent only on the state or history of those individuals. ${ }^{1}$ There is a long and productive tradition of developing statistical data analysis methods for such models. When individuals do interact, however, population models become dynamically non-linear. Non-linear dynamic relationships can lead to surprising population behavior determined more by who is interacting with whom in what way, than by the characteristics of individuals.

The difference between a collection of entities and a system is that in a system the arrangement of interactions between individual elements determines what the system will generate. In linearly dynamic population models, the population is treated as a heap of individuals analogous to a heap of sand. Just as switching the location of individual grains of sand does not change the features of a sand pile; it is assumed that changing the position of individuals in the population does not change population behavior. That is seen in the fact that for a typical data analysis the order of individuals in a data set makes no difference. Epidemiology is increasingly recognizing that populations are more than just the sum of their individuals and that epidemiologic analyses need to address causal factors that do not act strictly within or upon individuals. $^{2-5}$

Analyses in the standard plane, as shown in FIGURE 1, allows epidemiologists to use a wealth of computer data analyses packages. It also avoids the complexities of nonlinear dynamics. However, the assumption that the social network data plane of interactions between individuals, also shown in FIGURE 1, can be ignored is problematic for two reasons. First, the effects of individual risk factors can be wrongly assessed if in fact the connections between individuals cannot be ignored. Second, effects in the social plane might be ignored, when these could in fact be productively targeted in prevention and control efforts. 


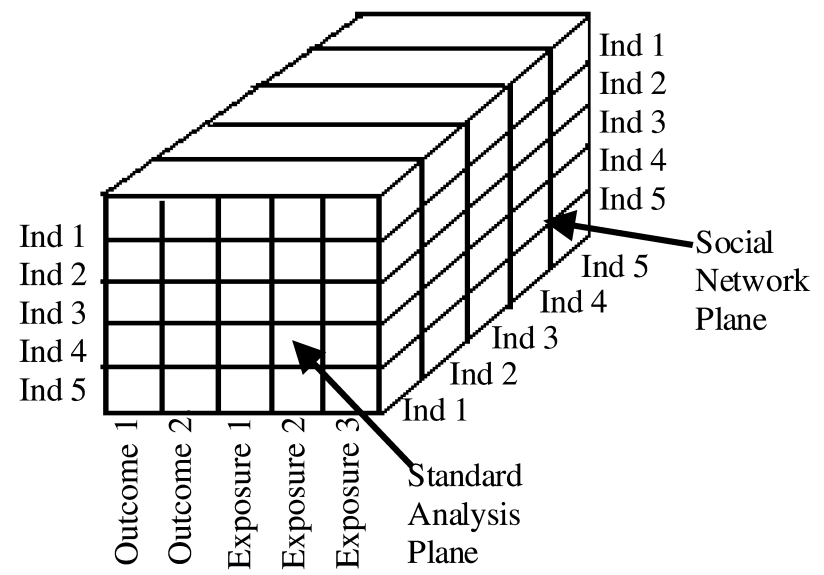

FIGURE 1. Planes of analysis for demographic and epidemiologic data.

Currently there is no easy and standardized way to gather and analyze epidemiologic data in the social plane. Epidemiologists often gather data in the social plane without treating it as a means to characterize the relationships between individuals. For example they collect histories of sexual interactions, data on geographical location as a risk factor for infectious disease, and data on social stress and social support. Usually these variables are not recognized as being relevant to the social plane and are "shoe horned" into the standard analytical plane. Methods to use data from the social plane to analyze transmission systems are becoming available for infectious diseases. ${ }^{6-9}$ However, the development of such methods is in its infancy and these methods are not much used by population scientists because a combination of powerful data and methods is not yet readily available. This paper outlines a path toward integrating the social plane by developing more flexible models and using phylogenetic data from organisms that reflect the history of transmission through the social plane.

The first step toward data analyses that integrate all relevant planes is to construct and analyze population system models that comprehend these plans. Although there is a long tradition among applied mathematicians and biologists of such model construction and analysis in infectious diseases,${ }^{10}$ it has been only rarely followed by epidemiologists. To move epidemiologists along this productive path, we see a need for a more comprehensive modeling approach that does not just stick to infection transmission system analyses. We see the need for a progression to models that generate data that epidemiologists can analyze, just as they analyze real world data. If this step is not taken, population system modeling from an epidemiologist's viewpoint is likely to remain an esoteric special undertaking. This is a major reason why we propose an approach to modeling that integrates a hierarchy of model forms that, when the right software becomes available, can be easily traversed by population scientists at the click of a mouse. 
Traversing the model hierarchy, we propose, should facilitate the pursuit of many goals, including the following:

1. Gaining insight into the behavior of population systems.

2. Generating and evaluating causal theory about population systems.

3. Facilitating the extraction of information from data, especially nucleotide sequence data.

4. Designing studies.

5. Enhancing policy decisions that affect population systems.

The model forms we discuss form a hierarchy in which one relaxes an assumption to transit to a higher model form. After the transition, new possibilities are opened up to generate models that capture aspects of reality that cannot be captured by model forms lower in the hierarchy. The hierarchy we present here is selected from many possible hierarchies involving the relaxation of many assumptions not addressed in this presentation. We have chosen to present a hierarchy based on relaxing three general model assumptions that separate four model forms because we feel these provide a structure widely applicable in population sciences. We label the four model forms as follows:

1. Differential equation, deterministic, compartmental models (DE)

2. Stochastic compartmental models (SC)

3. Individual event history models (IEH)

4. Dynamic network models (DNW)

The three assumptions relaxed in traversing this hierarchy are

1. Infinite numbers of individuals in each model compartment.

2. Homogeneity of individuals in compartments.

3. Instantaneous contacts with instantaneous and complete mixing after each contact.

Models toward the DE end of the hierarchy are easier to analyze. Models toward the DNW side of this hierarchy are more realistic. Each progressive model is constructed so that it can be formulated to give identical analytical results as its predecessor, but also so that it can be extended to capture aspects of reality that its predecessor cannot. The first two model forms (DE and SC) are the forms most commonly pursued by mathematicians or theoreticians, whose main concern is not the collection and interpretation of population data. Only the last two model forms, however, can generate data in the way data would be generated in field studies. They are, thus, the forms that can help population scientists design studies and interpret data analyses.

\section{GOALS OF DEMOGRAPHIC AND EPIDEMIOLOGIC MODEL ANALYSES}

Epidemiologists and demographers are more familiar with analyzing data than with analyzing systems by analyzing system models. Model system analysis assesses relationships between initial conditions, model parameters, and how variable values change over time. Mathematical model analysis establishes symbolic relationships between variables and parameters that hold in all states of the system. Numerical and simulation analysis lack such generality. Numerical analysis in this article refers only to using numerical methods to solve differential equations for a given set of parameter 
values and initial conditions. With such an analysis one only defines relationships for the specific starting conditions and parameter values examined.

Simulation analysis in this paper refers to Monte Carlo simulations of discrete events and discrete individuals in the highest three model forms. Monte Carlo simulations use random numbers to simulate chance as a determinant of events. In Monte Carlo simulation analysis, what happens to a population given a set of parameter values and initial conditions varies by chance so that multiple simulations are needed for a single set of parameters and initial conditions to assess the frequency distribution of different model outcomes.

Ideally model analysis and data analysis should be closely related processes. When this is the case, data analysis provides information about hypothesized causal processes in the real world rather than merely on relationships within data. The linking of model analysis and data analysis is less frequent in demography and epidemiology than in most other sciences. One reason for this is that epidemiologists and demographers are not trained in the techniques of causal model construction and analysis.

Demographic and epidemiologic models are usually models of populations or individuals in populations that experience events affecting the structure, size, health, and composition of the modeled populations. There are many reasons for constructing such models. We consider five.

\section{Modeling to Gain Insight into Population System Behavior}

Insight is often promoted by model simplicity. This is true whether the models are analyzed mathematically, numerically, or by simulation. An important way that simplicity promotes insight is by enhancing the possibility that mathematical analysis can find symbolic relationships that hold generally for a population. Numerical and simulation analyses provide insights only within the range of parameters and initial conditions that are explored.

Insight requires that the processes modeled be comprehensible. Although comprehensibility and simplicity do not have a one-to-one relationship, they are related. When a model includes realistic details that are not relevant to the phenomenon on which one seeks insight, model realism and complexity works against comprehensibility. On the other hand, models that are too simple and abstract to enable one to think about events in the model in the same way that one thinks about events in the real world may not facilitate understanding. For example, it is often hard to visualize how DE models reflect the individual events we think about in the real world because there are no such individual events in DE models. However, although DE models may be too simple and abstract to provide a framework for one's thinking about causal processes, they can facilitate insight by providing mathematical analyzability. Examination of functional relationships between model parameters and model variables over time, equilibrium values, threshold values, and growth rates can often provide insights that are not evident when using numerical or simulation analyses. When the focus is on equilibrium determinants or thresholds, simple stochastic compartmental models may also provide mathematical relationships; at the same time modeling discrete processes that are more natural to think about.

Consider the case of using infection transmission system models to gain insight on how the threshold phenomenon affects the distribution of infection in a population. 
Simple DE transmission system models with a single homogeneously mixing and homogeneous population with infection and immunity processes that involve simple time independent progression rates between states can be solved to yield the well known relationship ${ }^{10}$

$$
R_{0}=c \beta D \text {. }
$$

This expresses that the basic reproduction number equals the rate of contact times the transmission probability times the duration of infection. Many insights as to how social, genetic, and medical care factors relate to infection control have been gained by considering the above relationship. ${ }^{11,12}$ When mixing processes and infection and immunity processes are made more realistic, the relationships determining $R_{0}$ are far more complex ${ }^{13-17}$ and gaining insight from merely examining the formulæ becomes difficult.

Simplicity may not, however, always lead to the most appropriate insights. Simple models require radical simplifying assumptions that, if untrue, might lead to erroneous insights. How then, can one achieve a balance between simplicity and reality, where insights are useful? We suggest that the way to do this is to relax model assumptions. This makes it simple to test whether these assumptions affect the insight afforded by the model. To proceed in this way, one must be aware of the assumptions that generate simplicity and know how to relax them. Having a ready hierarchy of models that one can transit between at the click of a mouse should help in this regard.

\section{Modeling to Help Construct New Theory}

Although simplicity is the major modeling virtue for the pursuit of insight, logical completeness is the major virtue for construction of theory. To construct logically complete theory about population processes, one works out the implications for populations of fundamental elements and processes in a population. An effective way to work out those implications is to begin with more abstract and less realistic formulations and proceed then to relax the unrealistic assumptions used to make useful abstractions. Thus, to construct theory, it helps to have modeling methods that allow one to traverse model forms in a way that relaxes model assumptions and facilitates incorporation of more realistic detail.

The definition of fundamental in the above paragraph is relative. Every element and process can be broken down into component elements and processes. For example, contact processes in populations can be broken down into encounter processes, linkage processes, and behaviors within a linkage that facilitate transmission. Each of these elements could be further broken down. However, there is no sense in defining more fundamental elements and processes at either the individual or molecular level, or at system organization levels encompassing entire populations, unless one can generate usable theory from those elements. If one cannot construct usable theory from fundamental system elements, then by definition those elements are not fundamental to the task at hand.

Quite often, there may be no observations on the elements of a theory. For example, the standard model of subatomic physics encompasses details that, for many years, remained purely theoretical without any way to observe them. Nevertheless, the existence of models expressing standard subatomic theory stimulated new methods of 
observation. We see considerable potential for comparable stimulation of observation coming from theories of how encounter processes and linkage processes between individuals lead to population processes. Likewise we see value in constructing theory about how infection and immune processes at the cellular level in individuals affect infection transmission patterns in populations. If the theory is never constructed, the observations needed to evaluate the theory will never be made.

\section{Modeling to Better Extract Information from Data}

Epidemiologic data is rarely generated by processes acting strictly in the plane of analysis relating risk factor status of individuals to their disease outcomes. ${ }^{3}$ Much data, in fact, is better suited to reflect relationships in the social plane where many causal actions leading to disease occur. ${ }^{18-21}$ For example, place variables in infectious disease studies and courtship duration variables in sexually transmitted infection studies are better suited for reflecting who gets connected to whom than they are for defining individual risks. Since infection levels are often more responsive to who gets connected to whom as compared to risk factor frequency, developing ways to use this data in a more natural and effective way is important.

It is easiest to work out the effects on infection levels of patterns of who is connected to whom using DE models, but these have two deficiencies for extracting information from data on relationships between people. First, DE models are quite restricted in the connection patterns that they can generate. Second, they do not allow for the assessment of data analysis methods because they contain no individuals from whom data can be obtained. SC models do model discrete individuals, but they only count the number of such individuals in a model compartment and that greatly restricts the epidemiologic data sets that can be modeled. Thus, transition to IEH or DNW models is helpful when exploring ways to better extract information from data.

A new and powerful source of data relevant to infection flow is becoming available as the cost of studying nucleotide variation in infectious agents is decreasing. We will comment later on how models can be used to exploit such data to analyze infection transmission systems.

\section{Modeling to Design Studies}

Simple models for the sample size aspect of study design include models of the distributions of the statistics to be calculated from study data. The outcome assessed from such models is usually statistical power to detect an effect of a specified size. Many times the assumptions made in sample size calculations may be untenable so that even for this limited aspect of study design, one might want to use models to generate empirical distributions of the statistic of interest given different study designs.

Beyond sample size calculations there are many other aspects of study designs that need assessment. These include determining the sampling strategies that are most efficient, determining the biological or historical data that is most informative, assessing how the frequency of such data collection affects study objectives, and deciding who data should be collected from. In infectious disease studies, resolving these issues is complex and is usually done intuitively, but intuitions on these issues 
are highly fallible. A good modeling framework is needed both to better train such intuitions and to formally resolve the above issues for specific situations.

Because closed form solutions are not available to assess the above aspects of study design, their assessment is likely to be pursued through the use of simulations. An example is our current work to design vaccine trials for new vaccines against bacteria that cause ear infection in children. We want to know whether randomization of vaccine and placebo to individuals, families, or daycare centers will have more power and whether collection of culture material, blood for antibodies, and/or symptom data will provide more power for the money invested in the trial. We can use simulations of IEH or DNW models of infection transmission that incorporate alternative vaccine trial designs to help answer these questions. DE and SC models will not do because they do not generate the history of individuals. However, for initial selection of the ranges of natural history of infection parameters to examine, we use DE models to find parameter values consistent with observed infection patterns. To use those parameter values in IEH or DNW models, the models must fit into a hierarchy that makes it possible for higher-level models to behave exactly like lower level models under restricted conditions. That is to say that a hierarchy of models, such as we propose here, is needed.

Models can be used, not only to design sample and data collection, but to evaluate the analytical methods one will use from data collected from these designs as well. This is especially valuable for the analysis of infectious disease data since the analytical methods used on such data commonly make an assumption about independence of outcomes between individuals that is inconsistent with the nature of infection transmission. To assess how seriously one is being misled by a data analysis that makes an erroneous assumption, one can compare population system model parameters to data model parameter estimates made using data gathered from model simulations.

\section{Modeling to Enhance Decisions}

All decisions derive from models, whether or not those models are formalized and whether or not the decision maker is conscious of their model assumptions. Formalizing model assumptions can lead to more effective decisions and the avoidance of errors that arise due to ignorance of the assumptions one is making.

In constructing formal population process models to affect public or private decisions, the detail needed is often dictated by the nature of the decision. The model must include enough detail to capture the nature and consequences of different decisions. For example, when choosing between options for the control of Cryptosporidiosis, the model must include the processes involved in each control option and the outcomes that make a difference to a decision. If the control options are home water filters for HIV infected individuals versus water treatment plant improvements, the model must include HIV status, homes, water treatment plants, water treatment effects, and home filter effects.

The model must also include aspects that might alter the choice of interventions. An important aspect that could favor the choice of water treatment plant improvements over home water filters for the HIV infected is stochastically determined localized outbreaks set off by introduction of infection to a localized area that has been free of infection long enough for the level of immunity in the local population 
to fall. We have demonstrated that such effects of locality can be very strong and are missed by DE models. ${ }^{22}$ All of that makes for a fairly complicated stochastic discrete individual model.

Direct construction of such a complicated model is difficult because one must examine stochastic output in search of parameter values that generate realistic exposure and infection patterns. If one can work first with DE models to narrow the range of possible values, the stochastic simulation analysis undertaken will be more focused and productive. Once a model that is adequate to the task at hand has been constructed, there are a variety of ways it can be used to help make decisions. One could design computer experiments to determine cost/benefit ratios, cost effectiveness, relative costs of different interventions that achieve the same effect, ratios of effects given equal expenditures on alternative interventions, and several other approaches. Whatever the approach to these computer experiments, it will again be beneficial to narrow the space to be explored with an initial DE model analysis.

\section{A HIERARCHY OF MODEL FORMS}

The models we discuss here are hierarchical in the sense that each higher model form adds aspects of reality that the lower model form cannot use because that lower model form makes an unrealistic assumption that the higher model form does not. We discuss four model forms in such a hierarchy.

\section{DE Models}

At the base of the hierarchy are compartmental DE models that deterministically model continuous population segments. We consider here only compartmental models in which all inputs and outputs of compartments have the same unit structure, in our case populations of individuals. When individuals are the units of population, DE models assume that population size is infinite so that population size can continuously change to any positive value.

An example of one of the simplest possible DE models of a transmission system is the SIR model without vital dynamics, in which population is continuously divided into three states. The $S$ state contains the susceptible population. The $I$ state contains the infected and infectious population. The $R$ state contains the immune population. The only flows are from $S$ to $I$ and from $I$ to $R$ according to the following equations:

$$
\begin{aligned}
& \frac{d S}{d t}=-S \beta c \frac{I}{S+I+R} \\
& \frac{d I}{d t}=S \beta c \frac{I}{S+I+R}-\gamma I \\
& \frac{d R}{d t}=\gamma I,
\end{aligned}
$$

where $c$ is the contact rate per individual, $\beta$ is the transmission probability per contact, and $\gamma$ is the rate of flow from the infected to the immune state or the reciprocal of duration of infection. 
The course of a SIR epidemic, with $c=4, b=0.5$, and $\gamma=1$ is presented in FIGURE 2. Exploration of this sort of model leads to the insight that SIR epidemics peak when the fraction of susceptible individuals lowers the current reproductive number below one. It also reveals that epidemics end not because susceptible individuals are exhausted, but because there are not enough infected individuals left around to sustain transmission.

In a model of continuous population levels like this, there are no discrete individuals and no discrete events. The contact rate and transmission probability parameters represent processes that are analogous to contact between individuals and transmission during that contact when population size is large. Mathematically these parameters do not correspond to the discrete events that actually take place in populations.

\section{Stochastic Compartmental Models}

At the second level are SC models with compartments consisting of identical discrete individuals whose prior histories do not influence what happens to them. These compartments can be identical to the compartments of DE models with the difference that they relax the unrealistic assumption of infinite population size in every compartment made by the DE compartmental models.

SC models capture stochastic aspects of reality that compartmental DE models ignore. Accordingly, they model distributions of outcomes rather than just mean outcomes as DE models do. That makes the output generated by these models considerably more complex than the output generated by DE models. Dealing with this

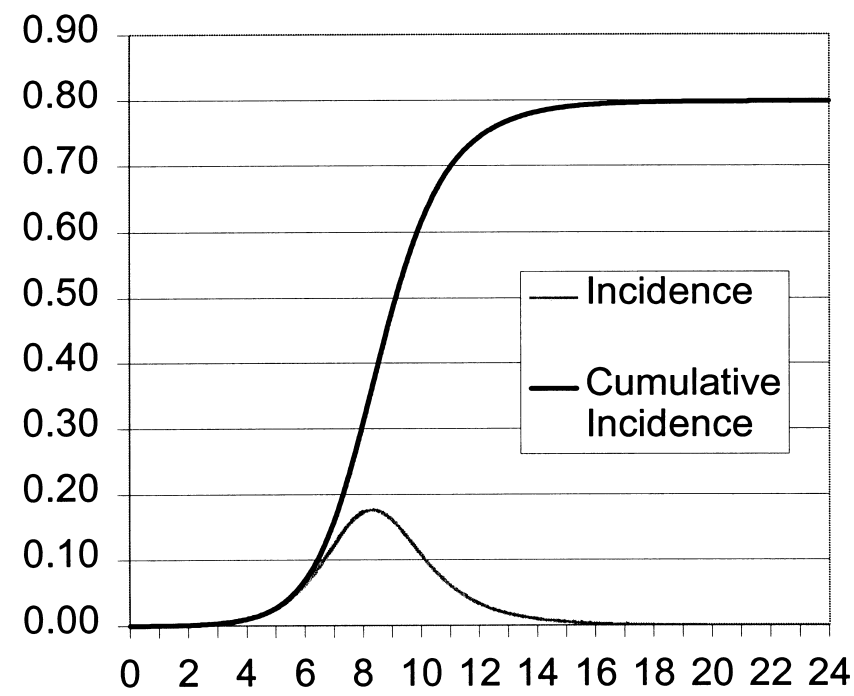

FIGURE 2. Incidence per month and cumulative incidence as a fraction of the total population in a DE model of a SIR transmission system without births or deaths, a contact rate of four per month, a transmission probability of 0.5 , and a duration of infection of one month. 
complex output and taking the time to generate it make SC modeling a more demanding task than DE modeling. Thus, there is a great advantage in proceeding as far as one can first with a corresponding DE model before advancing to an SC model form.

Consider now the SIR model, without vital dynamics, in a population consisting initially of nine susceptible individuals and one infected individual. An SC model of this population corresponding to the DE SIR model just discussed defines the rate at which the current state of the population flows into subsequent states, where one individual changes compartments. For example, with a current state of nine $S$ individuals and one $I$ individual the subsequent possible states are

- nine $S$ individuals and one $R$ individual (recovery event occurs)

- eight $S$ individuals and two $I$ individuals (infection even occurs)

In our simple SIR model there are only two possible events. These are new infection or recovery from infection with acquisition of immunity. Thus there are always only two possible classes of next events. When flow into state 1 occurs, there will be no further flows since there are no infected individuals to infect the susceptible individuals. When the flow is to the state 2 , then the population can flow next into one of the following two states:

- eight $\mathrm{S}$ individuals, one $I$, and one $R$ (recover event occurs)

- seven $S$ individuals and three $I$ individuals (infection event occurs).

Which flow occurs and when that flow occurs is a matter of chance. Thus, at any one time, there are different probabilities for various model states. A full SC model analysis would establish the probability of each model state at each time. For example, one second after the initial state there is some extremely small chance that all 10 individuals are in the $R$ state. Indeed there is some chance that the population could be in every possible combination of $S, I$, and $R$ that sum to ten. An SC model defines the probability of each of those states over time. Thus, SC model output is considerably more complex than DE model output. For very simple models like the one just presented, it is possible to write DEs to describe the probabilities of each possible state over time. However, only modest increases in population size or model complexity quickly make this an impractical task. Thus, the way that SC models are commonly analyzed is through simulation.

If the compartments of an SC model correspond to the compartments of a DE model, then as population size in the SC model increases, the mean behavior of the SC model progressively approaches that of the corresponding DE model. At small population size, however, the mean behavior can differ quite significantly due to stochastic events. The mean behavior of the DE model differs from the mean behavior of the SC model because in the DE model, the state of having no infected population cannot occur, whereas it can and does occur in the SC model. When it does occur in the SC model, the fact that no subsequent new infection can occur causes the mean $\mathrm{SC}$ model behavior to differ from the DE model behavior.

Often the analysis of SC models will be through simulations rather than through mathematical analyses. To insure that SC model simulations behave like DE models in the sense that the future state of the SC model is only influenced by the current state of the model and not by past events, one can simulate just one event at a time. 
The time when that event occurs can fall anywhere on a continuous time scale. To simulate the single next event, the rates of all potential next events are summed to give a single overall rate for the next event. For example in our SC model of an SIR process with one infected individual and nine susceptible individuals, the rate that any susceptible individual will be infected and the rate that the infected individual will lose their infection and gain immunity are summed. From that sum the cumulative probability distribution of times to the next event is determined. Then a specific time for the simulated next event is selected by drawing a random number from the zero to one and ascertaining the time, in the cumulative distribution of times, to next events corresponding to that randomly drawn number. Each event has a probability of selection proportional to the rate of that event. The next simulation step is to determine whether the next event is a new infection or a recovery from infection.

An SC simulation conducted in this manner realizes a single instance of the stochastic process. For a more thorough analysis, enough simulations should be performed to outline the probabilities of different model states over time. Generally that means hundreds of simulations. Thus, SC model analysis takes much longer than DE model analysis. The more complete and realistic output generated also means that it takes more time to interpret the results.

Note that to be realistic and to correspond to the $\beta$ and $c$ parameters in our DE model, the new infection event could be broken down into a contact event and an infection transmission event. The rate of contact events for each individual is $c$. The rate of new infection events is the sum of the rates of contact events by each susceptible individual multiplied by the probability that the contact is with the infected individual multiplied by the probability that infection is transmitted during that contact. To correspond to the DE model process, the probability of the next event is calculated using the assumption that, immediately after having made a contact with one individual in a compartment, the probability that the next encounter is with that individual is not influenced by just having contacted that individual. In real geographic and social space, this would be an unrealistic assumption. We do not relax that assumption until we reach the fourth level (DNW) in our model hierarchy.

Note that, in this SC simulation process, it is not necessary to determine which of the nine susceptible individuals becomes infected. The model output is the number of individuals in each state, not which individual is in each state.

\section{Individual Event History Models}

At the third level are individual event history (IEH) models. These are models of individuals just as are the SC models. SC models only count individuals with different characteristics. They do not record the history experienced by each individual. They require that all of the different combinations of individual characteristics that are possible be defined ahead of time and integrated into the model structure. Individual event history models do not have this requirement and they do record individual histories. That makes them more appropriate for simulating data that might be collected in an epidemiologic study. It also means that they have even more detailed and complex output than the SC model, which takes more computer time to simulate and, if the history of each individual is considered, more time to digest and interpret the results. Conceptually, however, the contact and infection transmission processes in the SC and IEH models are identical. 
Since IEH models need only define processes that individuals experience and do not need to define ahead of time all the possible classifications of individuals, the first step in their construction has traditionally been the construction of a population of unique individuals. One problem with this approach is the difficulty in analyzing how different possible combinations of individuals and individual interactions in a model affect its behavior. It is difficult and very time consuming when starting directly at this level to determine whether one is adding unnecessary detail and complexity to the model structure.

Within the hierarchical approach we advocate here, the first step is construction of an SC model. There are then two reasons for transiting from the SC level to the IEH level. The first is that one needs individual event histories in order to evaluate a study design or data analysis approach. The second is that, as more characteristics of individuals and events that they can experience are added to the population model, the number of compartments that need to be predefined rises exponentially. This can rapidly generate huge spaces that consume computer time and memory. The IEH avoids this problem by following individuals rather than compartments as the SC model does. This means, however, that the size of populations that can be modeled with an IEH approach is more limited by computational needs than is the case for SC models.

Because IEH models follow individuals rather than compartments, modeling multiple interacting agents with cross-reactive immunity between them becomes possible. Another model elaboration possible in IEH models, but not in SC, is that one can use continuous measures of characteristics. For example in an SC model one might have three levels of susceptibility and four levels of different contact rates that individuals can experience. In IEH models, one can define continuous distributions for these two characteristics. The characteristics assigned to any particular individual in simulations of the model can be assigned by randomly choosing values from these distributions. This makes the computer simulation costs of adding new dimensions and categories of individuals less for IEH than SC models. In order to decide at what level of complexity the transition from SC to IEH models is justified, it would be quite useful to be able to compare the performance of simulations of each model form. Software that facilitates the transition between model forms would greatly facilitate such comparisons.

At the IEH model level, one can add many details of individual characteristics. One could add geographic location detail for example that could then be allowed to have important effects on who contacts whom in the model. Even within the context of instantaneous contacts, one could in this way add many realistic aspects to the contact process. Geographic or social location could be used to define fixed links between individuals in the model. The one aspect of contact processes that cannot be added, however, is the formation of new ongoing links between individuals. For that, transition to DNW models is needed.

\section{Dynamic Network Models}

The first three hierarchical models all make the assumption that contacts between individuals are instantaneous events with no duration in time. They also assume that contact events do not influence who will be contacted in the future or the future rate of contacts. Dynamic network (DNW) models relax these unrealistic assumptions. 
In so doing, they generate networks of connected individuals that are continually changing over time. An example of a DNW model that we have analyzed to show its consistency with a DE model is a gonorrhea model we called GERMS. ${ }^{23}$ This model has a process through which individuals encounter each other that is identical to the contact process discussed for the SC and IEH models. The difference is that in an SC or IEH model, encounters lead instantaneously to short contacts that in fact have no duration in time. In a DNW model, on the other hand, encounters lead to a linkage between two individuals. Contact events that can transmit infection only occur between linked individuals in DNW models. That generates a strong dependence between who is contacted at one instant in a fashion that can lead to transmission of infection and who is contacted in this way at the next instant.

Computer simulation of DNW models is more involved because the state of linkage of every person to every other person must be maintained and because there are separate encounter, linkage, and contact events to be simulated. The potential complexity in the detail of the output is also greatly increased, just as it is in an SC to IEH transition. Even if one wants only identical output to that examined at the SC or IEH levels, there is more data that must be summarized before one can generate such output. For example we used our published DNW model called GERMS to compare the ability of DE and SC models to capture important effects of geography. ${ }^{22}$ The simulations were very slow and we did not have the flexibility we desired because we were using a simulation fully capable of simulating a DNW model to simulate an SC model. We did this by setting the duration of linkages extremely short and grouping individuals into compartments.

If one is examining sexually transmitted infections where the pattern of ongoing linkages between individuals can be highly influential, ${ }^{24}$ one will want output that goes beyond the output desired for SC and IEH models. For example, one might want output that describes the level of concurrent linkages since that has been shown to be important. ${ }^{20,21}$ One might also want network measures that assume a connection from one linkage to another even when the linkages are not concurrent but the timing of linkages is such that one might carry infection from one linkage to another.

A DNW model is still one where the next state of the model is only dependent upon the current state of the model. The difference is that now there are new states of the model defined by who is linked to whom.

\section{General Aspects of This Model Hierarchy}

The transition between the SC and IEH level models is different than the transition between DE and SC or between IEH and DNW. With regard to these latter transitions, there is no way that models at the lower level can be elaborated to correspond to all of the models at the higher level. In contrast, the homogeneity within compartments assumption of SC models can be relaxed just by adding more compartments to the SC models until they approach the structure of IEH models. Nevertheless, the simulation of these two model forms is still hierarchical for two reasons. First, simulations of SC models do not retain individual histories, whereas individual event history models do. Second, to simulate an SC model, all the states into which individuals can be classified have to be enumerated and entered into the model simulation in advance. That is not the case for individual event history models. 
These four model forms have been constructed many times for many different types of system analyses. Usually, however, they are constructed in isolation and in a manner that does not allow for transition between model forms. Special care must be taken in model formulation to allow for these transitions. A disconnect between DE compartmental models and models with discrete individuals is created when simulations of the models with discrete individuals are formulated using discrete time steps. Another disconnect is generated when long lists of future events are used in the simulation as is commonly the case with $\operatorname{GPSS}^{25}$ as well as other simulation packages. This disconnect arises because the occurrence of one event on the list can alter the conditions that might lead to subsequent events on the list. A further disconnect is created when contact structures in discrete individual models are formulated as fixed networks. The use of fixed network structures is the most common way to formulate network models. However, to be consistent with the assumptions of lower level models, network links must be dynamic enough so that they can be short enough to have no practical consequences.

In order to maintain the ability to transit between model forms, there are two restrictions we have accepted with regard to model construction. We use only continuous time and we formulate all distributions of times to next events as Erlang distributions so that they can be handled by a series of memoryless compartments so that the future states of the model are fully defined by the current state.

Benaim and Hirsch have mathematically proven the identity of compartmental DE models and SC models when the population size of the SC models is allowed to go to infinity. ${ }^{26}$ Vlad and Schonfisch have mathematically proven the identity of DNW models and DE compartmental models when both population size and the rate

TABLE 1. Characteristics of four model forms

\begin{tabular}{|c|c|c|c|c|}
\hline & DE & $\mathrm{SC}$ & IEH & DNW \\
\hline Assumes infinite population size & yes & no & no & no \\
\hline $\begin{array}{l}\text { Assumes homogeneity within compart- } \\
\text { ments }\end{array}$ & yes & yes & no & no \\
\hline $\begin{array}{l}\text { Assumes that contacts have no duration } \\
\text { in time and that who is contacted is not } \\
\text { influenced by history of prior contacts }\end{array}$ & yes & yes & yes & no \\
\hline Contact rates can be defined by region & yes & yes & yes & yes \\
\hline $\begin{array}{l}\text { Allows one to evaluate study design } \\
\text { and data analysis methods }\end{array}$ & no & partially & yes & yes \\
\hline Models events deterministically & usually & rarely & rarely & rarely \\
\hline Models events stochastically & rarely & usually & usually & usually \\
\hline Ease of mathematical analysis & easiest & OK & hard & harder \\
\hline Solved numerically & yes & no & no & no \\
\hline Usually simulated for analysis & no & yes & yes & yes \\
\hline Complexity of analytic results & simplest & $\mathrm{OK}$ & complex & $\begin{array}{c}\text { most } \\
\text { complex }\end{array}$ \\
\hline
\end{tabular}


of change of network links go to infinity. ${ }^{27}$ Theoretical identity, however, could be lost by the vagaries of coding complex systems for computer simulation. Having a model form at a lower level whose behavior is well established provides a validation for the coding of a model at a higher level.

The assumptions and other aspects of the four model forms are summarized in TABLE 1. Note that it is possible to have compartments in DE models correspond to geographic and social regions. Thus, geography can be integrated into each of the four levels in an identical fashion. That does not mean that identically formulated geography will have the same effects in each model form. We have shown this not to be the case. ${ }^{22}$

Two advantages of analyzing a population system using these linked model forms as opposed to using any single model form that cannot be linked to others are:

- The ease of performing analysis of lower level models helps focus the analysis of the more complex higher model levels.

- Simulation code for the more realistic model forms can be validated by checking consistency with lower level models under restricted conditions consistent with the assumptions of those lower level model forms.

\section{Other Possible Hierarchies}

Other model forms that further elaborate this hierarchy are possible. For example:

- DE models could be elaborated with partial differential equations across age and time. Age of individuals in a population is otherwise a model elaboration that would not be undertaken until the IEH model level is reached.

- DE models could have compartments for pairs of individuals in the tradition of models by Dietz and Hadeler. ${ }^{24}$ This relaxes the assumption about complete and instantaneous mixing that is otherwise relaxed when one transits to DNW models.

- Models at any level in the hierarchy could have compartments for the medium through which infection is transmitted, such as water for waterborne diseases. If one needs to choose between alternative interventions that protect water sources from contamination, treat contaminated water, or improve the protection from contamination in the water distribution system, one would need to explicitly model the water medium as it goes through different phases.

- Models at any level of the hierarchy could include behavioral aspects of individuals that change either their activity in making contact with others or their availability to be contacted by others.

The hierarchical structure we have outlined would still form the backbone of these more complex hierarchies. 


\section{A CRYPTOSPORIDIUM TRANSMISSION EXAMPLE}

An important question posed by the Environmental Protection Agency of the United States is: what will be the effects of different interventions to stop the waterborne transmission of Cryptosporidia? This parasite causes considerable disease both in epidemics and endemically. It escapes standard water treatment methods. In individuals with weakened immune systems it is often fatal. Population level analyses of nucleotide sequences from Cryptosporidia have demonstrated that there is a strain of Cryptosporidia transmitted primarily from human to human and another strain transmitted largely from cows. ${ }^{28,29}$ If it is assumed that very little human-tohuman transmission takes place, the human infection transmission system can be disregarded. In this case, cost-benefit analysis demonstrated that it would be more cost-effective to put water filters in the homes of HIV patients than to put ozonization procedures in water treatment facilities. However, for the human strain where the agent is sustained in the population by transmission from human-to-human via a variety of routes including water, the issue is less clear.

Water could play a variety of different roles in a transmission system. It might be the key mode of transmission sustaining the agent in a population. In that case one could get rather dramatic effects if one could eliminate sufficient waterborne transmission to stop circulation. On the other hand, other modes of transmission might be sufficient to sustain the circulation of Cryptosporidia, but water could amplify that transmission. All of the five goals of transmission system analysis we outlined earlier can be pursued to address this case of waterborne Cryptosporidia transmission.

\section{Insight into the Population Dynamics of Cryptosporidia Transmission}

Examination DE models can provide insight into how, why, and to what extent waterborne transmission might increase infection levels in a population. We have conducted such an analysis and found that the effects of water contamination on endemic infection levels are pretty much what one would expect from the increase in the overall basic reproduction number $\left(R_{0}\right)$ in the theoretically derived relationship $P=1-1 / R_{0}$, where $P$ is the endemic prevalence. ${ }^{30}$ Thus, waterborne infection makes its greatest contribution to endemic prevalence when other modes of transmission generate an $R_{0}$ close to one.

DE models, however, disregard stochastic effects arising when infection dies out in a localized population. Likewise, they miss the effects of chance introduction via water to a population without infection. Cryptosporidia immunity is lost relatively quickly, so that when populations stay free of infection for some time, their potential for epidemic spread upon introduction of infection via water grows quickly. Given this scenario the indirect effects of quite infrequent transmissions via water could be very great and water treatment at the plant that could prevent such introductions would be justified.

In order to gain insight into the extent and the conditions under which such introduction might be important and the extent to which DE models will miss such effects, we compared very simple DE models to SC models in which the population sizes were made realistically small. ${ }^{22}$ The intent of this model analysis was not to be realistic, but to provide insight regarding the conditions where stochastic die out and 
introduction of infection could be important and how big such stochastic effects could be. Some of the insights provided are reviewed below.

Insight on Spatial Effects Missed by DE Models

Since Cryptosporidia immunity is not long lasting, we model its natural history of infection and immunity by adding a flow from the $R$ state back to the $S$ state to the SIRS model. To capture the effects of space, we model 100 population subgroups arranged in a circle. The population in each subgroup mixes with others at a site associated with their subgroup, at sites associated with each of their neighbors, and at a site where all subgroups mix. The central site is a disseminating site connecting distant individuals just as water could for Cryptosporidia. The purpose of this model is to gain insight into spatial effects and how deterministic and stochastic models capture them. The pattern of subgroups and the places they mix in this model is illustrated with a reduced number of subgroups in FIGURE 3.

In FIGURE 4 we examine the endemic prevalence values generated by this structure when parameter values are set so the basic reproduction number is two. We compare the behavior of a DE model having this contact structure with an SC model where each subgroup has only 12 individuals. When all the mixing is in the central

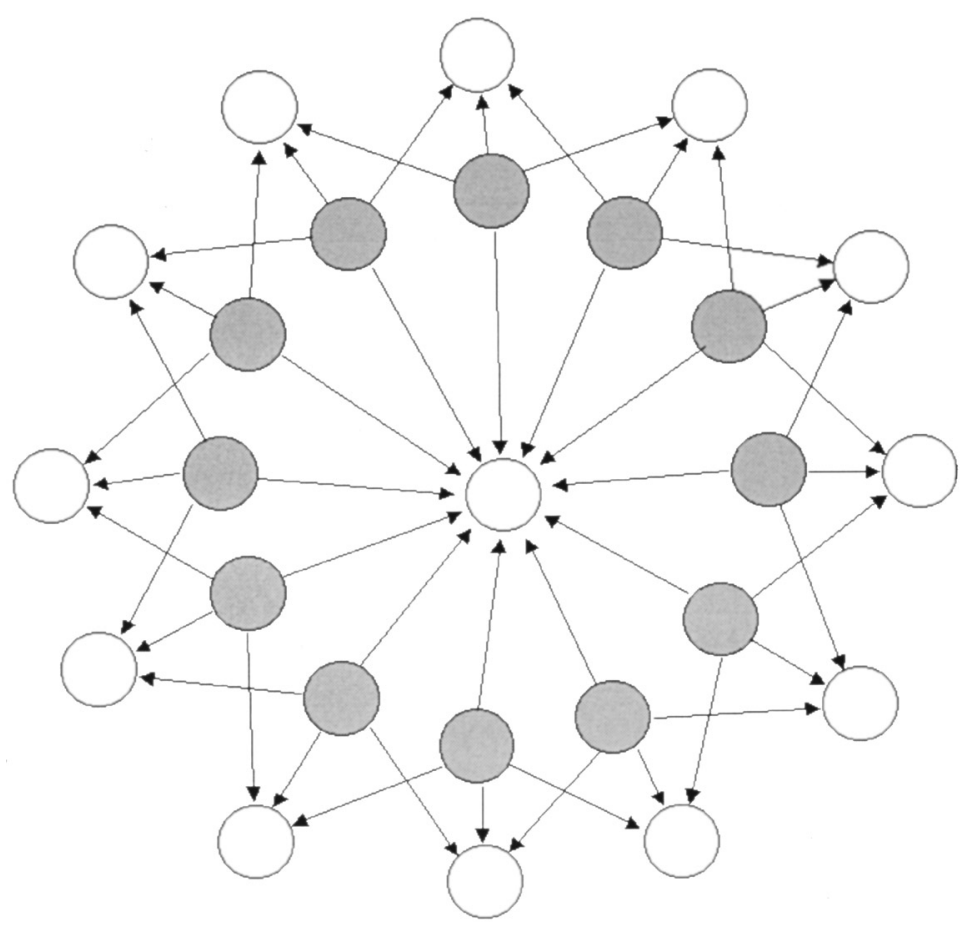

FIGURE 3. Contact structure in the models with 100 groups and an SIRS pattern of infection: $\bigcirc$, mixing site; $\bigcirc$, compartment of individuals or popuation segments. 


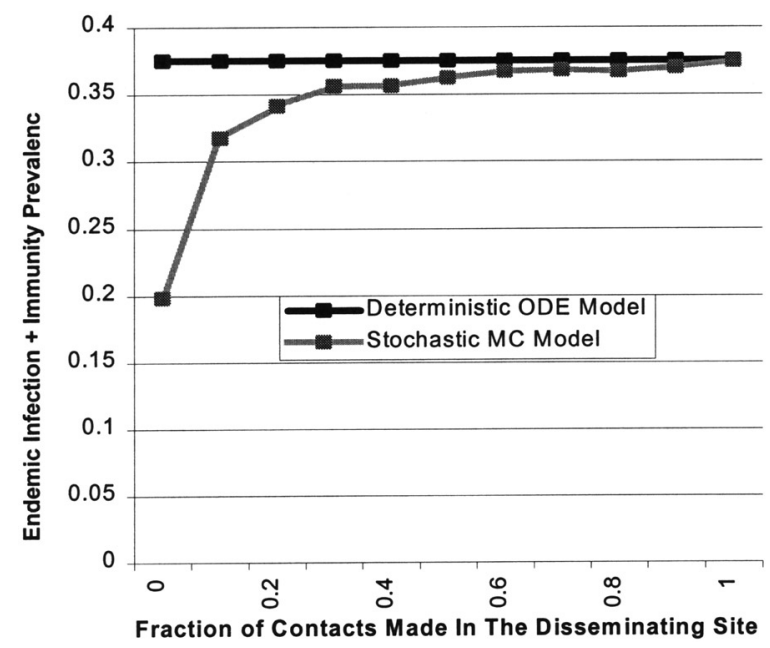

FIGURE 4. Comparison of endemic prevalence of infection plus immunity for deterministic and stochastic models where the fraction of contacts made in the central disseminating site is varied.

disseminating site, we see that the endemic levels generated by the two model forms are about the same. Of course in the stochastic model the levels are fluctuating, whereas in the deterministic model they are steady. As more of the mixing is local, however, we see no effect in the DE model but in the SC model the endemic infection level drops. Detailed examination of the results reveals that this is due to infection temporarily dying out of local parts of the model until by chance there is a new introduction.

We demonstrated that differential equation models fail to detect differential effects of local versus disseminating contacts. Consequently, the deterministic models considerably underestimate the role of disseminating transmissions like water and overestimate those of local transmissions that are more likely to be person to person. That is because the differential equation assumption of either infinite population size or divisible humans means that no contacts are more local or disseminating than any other contacts. All contacts can reach out to an essentially infinite distance. The failure of differential equation models to capture the effects of local versus disseminating contacts can also be explained as a failure to capture the effect of local die out of infection. Given a basic reproduction number greater than one, all such die out is due to stochastic events. In a deterministic model, there can be no such die out.

Further examination of this model shows that DE models increasingly miss disseminating transmission effects as the period of immunity increases so that the chances of local die out are increased. It also shows that DE models underestimate the effects of interventions to control disseminating transmissions. As models of Cryptosporidia transmission are made increasingly realistic, these effects captured by stochastic models but missed by deterministic DE models increase. 


\section{Developing Theory about Cryptosporidia Transmission Systems}

Now that it is clear that humans maintain the circulation of human strains of Cryptosporidium, the question becomes: "how important is transmission via water and via direct contact or other non-waterborne transmission modes?" It is time to develop reasonable and testable theory about the role that waterborne and direct person-to-person transmission play in transmission systems.

The way to develop such theory is to explore the behavior of models that have elements of a system that might represent reality. The first order of business here is to construct a transmission system model that allows one to explore the system conformation determinants of direct and indirect effects of water transmission and direct person-to-person transmission. Exploration of such a model should help one decide which theory might be reasonable or unreasonable. It should also help one to organize what is known and unknown about the transmission system so that one can better identify areas where new theory is needed. Most of all, it should help organize one's thinking about the nature and behavior of the transmission system.

This process could begin with DE models. That would facilitate wider explorations. Insights gained from the comparison of DE and SC models presented above indicate that an exploration purely at the DE level has a high chance of missing important aspects of transmission systems related to stochastic effects. To develop theory about how levels of personal hygiene and population mobility alter the effects of water on population infection prevalence, one would want to explore SC or other stochastic models, such as IEH and DNW models after initial exploration with DE models.

Just developing any possible theory does not generate the excitement of new discovery as much as does the exploration of the real world to see if the theory is correct or if it represents reality better than some alternative theory. So the development of theory should go hand in hand with developing ideas about data relevant to the theory that can be gathered in the real world. In our Cryptosporidia example, models are essential for conceptualizing new forms of data and developing new ways to use that data.

\section{Facilitating the Extraction of Information from Data}

Once a reasonable model of the transmission system is constructed and hypotheses are developed concerning the role of water transmission, the next task is to determine what data can help us refine our transmission system model, estimate its parameters, or test our hypotheses. The standard approach to exploring data needs in epidemiology is to calculate required sample sizes, using the assumption that all individuals are independent and that there are no connections between them that make the population behave as a system. Clearly this approach will not work where our hypotheses have to do with the conformation of a population system. We need to develop new approaches using models from levels three or four that specify longitudinal data for each individual in the simulation.

Statistics relevant to transmission systems are quite undeveloped. Models are needed to help investigators think about what statistics might be useful and then to explore the properties of the various statistics proposed. How this process proceeds 
will depend upon the type of statistics that are proposed. We see three broad categories of data and corresponding statistics:

1. Empirical data for which the development of statistics does not require the use of a causal model of the transmission system,

2. Causal system data that requires a causal system model to choose parameters to be estimated, to develop estimation procedures for these parameters, or to formulate indices reflecting causal system states,

3. Phylogenetic data on organisms isolated from individuals with known contact points in the transmission system.

\section{Empirical Data}

Two examples of empirical data come from study designs that are currently being implemented. In one study, the frequency of Cryptosporidia is examined in populations served by ground water sources and by surface water sources. ${ }^{31}$ The surface water sources have much more Cryptosporidia contamination than the ground water sources. In a second study currently under way, families are receiving either a real or a placebo water filter apparatus in their homes that eliminates Cryptosporidia in the household. With such empirical data, one examines correlation between exposures and outcomes and calculates risk ratios, odds ratios, or risk differences.

An issue with such data is, which statistics can be calculated and what do different values of those statistics imply about the issues being addressed. With these two study designs, the statistics seem rather straightforward. Average infection levels in towns with different types of water supply or families with real or placebo water filters seem like reasonable outcome statistics. Averages might be compared with differences or ratios. Median values might also be compared in these ways. To choose among the statistics used to extract information from empirical data, one could construct a wide variety of causal models at the IEH or DNW levels. In such models, one knows exactly what is happening in the models. This enables one to see which statistics best reflect what one is interested in learning from the statistics.

In the example from the community and family study designs mentioned earlier, one might want to see which statistics are most predictive of the effects that reducing water contamination might have in a population. Such an exploration is likely to reveal that many times the family design statistics will show little effect of water in situations where improved water treatment could have dramatic effects. That is because those statistics only reflect direct effects of exposure and not indirect effects arising from such things as the rare introduction of infection via water into a neighborhood that is free of infection and has built up high levels of susceptibility to infection as immunity has waned.

\section{Causal System Data}

Usually one is doing a study to learn something general that is not unique to the study population. Extrapolation of empirical results to new populations is not supported by sound theory. To get such support, one must seek data that informs one about the validity or the parameter values of causal models, such as infection transmission system models. 
Data for a transmission system analysis includes system outputs such as patterns of infection by age, region, or mixing group participation. Such data is similar to data appropriate for standard epidemiologic analyses. Exposure data, however, differs quite significantly from the usual sort of data used to examine associations between exposures and diseases in a population. It is not just data on individual exposures. It is data on the interactions or relationships between individuals in the transmission system that allows one to make inferences about the population conformation of such contacts. That is to say, it is data in the social plane discussed previously that is ideally analyzed on the basis of the overall pattern of connections. Transmission system models need to be explored to determine how such data can be used most efficiently. Since the appropriate use of such data is in its infancy, much of what we say about it here is speculative.

Such data might allow one to calculate how intensely individuals make contact with certain mixing groups and how many individual represent bridges between different mixing groups. For example, one might examine contact patterns in daycare centers and schools and determine how many individuals represent potential bridges between different centers and schools, either directly or through contacts they make in their household. Using such data, one might develop indices relevant to contact pattern models reflecting the intensity of contact clustering and the extent of linkages between clusters. These then might either be empirically related to outcome measures.

In the absence of waterborne transmission we might expect the following empirical association of contact patterns and system effects. Populations with clusters of isolated contacts are likely to have a transmission system that does not efficiently sustain transmission at the population level. Populations where individuals mix widely and are not clustered will have higher levels of infection. Those with wellconnected clusters will have the highest levels.

Empirical use of such data would just allow one to examine the correlation of infection levels in a population with contact patterns in that population. A causal model approach would use this data to estimate contact parameters in a transmission system model. This might be done by directly estimating the rate at which individuals in different subpopulations contact each other. Or it might involve finding the contact parameters in transmission system models that could explain observed patterns of infection in the population. It is the use of data in a causal system model rather than any intrinsic aspect of the data that makes it "causal system data". Having software that enables one to transit between model forms should greatly facilitate getting to the point where such causal system data can be analyzed using causal system models.

\section{Phylogenetic Data}

Data on incidence or prevalence of infection in different subgroups may not be of a sufficient complexity to provide information on a system as complex as a transmission system. A much more complex form of data that reflects not only immediate relationships in a transmission system but also past relationships is phylogenetic data from the nucleotide sequences of organisms isolated from individuals with known contact points in the transmission system. Phylogenetic data obtained at one time, without data on the contact points of infected individuals in the transmission 
system, has been used to characterize population dynamics of infectious agents. 32,33 Because infectious agents evolve rapidly, it is possible to use sequence data collected over time and from individuals with known contact points in the transmission system. Data with these two aspects can more powerfully and completely define population dynamics than can data from one time point without histories of contact.

Such data lies in the social plane and does not fit standard epidemiologic methods. The format for phylogenetic analysis is identical to that for social network analysis. Individuals are arrayed in both rows and columns and table entries express degree of connection. When organisms recovered from individuals in a population are sequenced, the array would have one level for each nucleotide. These multiple levels can be reduced by a phylogenetic analysis that determines tree arrangements and phylogenetic distances.

Such data is valuable because transmission events represent bottleneck events in the population history of an infectious organism. Such events can fix nucleotide sequence variation in a population. Consequently, the phylogenetic tree reflects the pattern of transmission that connects individuals. Just as one can trace a transmission back to a common ancestral source for two individuals, a phylogenetic analysis assesses the distance between two individuals by tracing an inferred tree back to common ancestors.

Statistics to use this sort of data for transmission system analysis are not yet developed. We see many possibilities, however. We feel that both empirical analytical approaches and theoretically developed analytical approaches should be pursued. Consider first a more theoretically based approach to resolving important issues relevant to Cryptosporidia transmission systems.

In an IEH model of a Cryptosporidia transmission system, additive phylogenetic distance models can be used to characterize the paths of infection generated by repeated simulations. These additive distance models can characterize the positions on a phylogenetic tree of organisms from people who reside in different neighborhoods and use different water systems. The form of infection transmission trees is identical to the form of phylogenetic trees. The phylogenetic trees of the organisms reflect the transmission trees. A theoretically based analytical approach would involve fitting model parameters to generate transmission trees that fit observed phylogenetic trees. Just as model based approaches to estimating phylogenetic trees are computationally intensive, this approach may also overwhelm our computational capacity.

However, phylogenetic analysis has made great progress using empirical approaches that are less computationally intensive. The same path might be the best one for a science of transmission system analysis. Various statistics on the shape of the transmission trees and on the clustering of organisms from individuals with different exposures might be calculated from models where water does and does not play a key role in the population level of infection. The distribution of those statistics can then be compared to observed phylogenetic trees to help infer whether those trees come from situations where water is key or situations where water does or does not play a key role. For example, one statistic from a prospective study might use sequences of agents isolated from water and sequences from agents isolated from individuals in the same population who do or do not drink the water. The average phylogenetic distance of agents from individuals who drink the water to agents 
isolated from water in previous time periods should be less than the distance of the agents isolated from individuals who do not drink the water. How distance patterns are related to the role that water plays could be explored in IEH simulations that provide a structure on which evolutionary models are superimposed.

\section{Designing Studies}

Consider the studies of populations with surface and ground water. Models could help determine the sampling designs within or between communities that could most effectively detect water effects. Issues that could be addressed are

1. whether individuals in large families or small families should be sampled,

2. the age ranges that should be sampled,

3. the sizes of samples in a community versus the number of communities sampled, and

4. which type of communities should be sampled:

4.1. large versus small communities,

4.2. unsanitary versus sanitary communities, or

4.3. isolated versus highly connected communities.

Another set of issues to be examined through model analysis deals with what data should be collected and what new biological data collection methods should be developed for the study. For example, one could compare the efficiency and validity of studies using stool agent identification, serological infection detection, or merely reporting of symptoms. Whether sera or stools are examined, one should use models to determine how the sensitivity and specificity of the assays used affect study efficiency. One should also explore how the use of methodologies to distinguish human strains from animal strains would affect the efficiency of the studies.

\section{Enhancing the Decision Process}

Given the great number of unknown parameter values and the difficulty in testing the theory on which Cryptosporidia transmission system models are constructed, the decision process for designing interventions to control Cryptosporidia transmission cannot be pursued by optimizing control strategies in a model of the transmission system. Models can, however, be used to enhance choices between two intervention alternatives.

In the case of Cryptosporidia, a current pair of choices is whether to put new ozonization processes in water treatment plants or put home water filters in the homes of HIV patients. A model to examine this choice needs data about family structure and direct contact structure between families. It also needs data relating to contamination of source water by infected individuals, take up of such water into water treatment plants, the water treatment process itself, and consumption of water from home and other sources. In other words, the model requires a fair degree of detail. Otherwise it will not comprehend the two alternative choices.

A first step in developing a model with the needed detail might be to construct a DE model that does not contain family structure, but includes family transmission with other levels of not-waterborne transmission. Using such a model should help one establish some of the general characteristics needed for the model and to get an idea what ranges parameter values might have. 
A second step would be to construct an SC model that implements the stochastic discrete individual equivalent of the DE model. A third step would be to construct and analyze the simplest SC model that can comprehend the two decision alternatives. This model would add families to the previous SC model, but it would not distinguish individuals by age or by different roles in families. Analysis of this model would consist of defining a boundary region in parameter space that separates the two decisions under consideration. To do that, a very clear decision criterion is needed. In a population model without age or without varying severity of illness, that criterion can use neither the age of infection nor the total severity of illness. It will have to be confined to number of infections over time. Time specificity is still important in the criterion because one decision might lead to a faster fall in infection rates than the other even though the eventual equilibrium effects might be greater given the decision that induces the slower fall.

If the region that comprehends one of the decisions is judged to be so far from reality that the other decision is clear, the process might end with no precise boundary area being defined; the boundary only having been determined sufficiently to conclude that one decision does not include a realistic parameter space. In this case there might be no apparent need to relax unrealistic assumptions in the model or to elaborate the model to comprehend a more detailed decision criterion. Of course, there is no guarantee that an even more realistic model cannot overturn any degree of effect. However, decision making of this sort is not an exact science. Model assisted decision making is only useful if it leads to effective action and does waste too much time being paralyzed by alternatives or in constructing models.

On the other hand, if the decision maker does not like the criterion that is used in the simplest model, or thinks that the unrealistic assumptions of the model make it unconvincing, then the model should be elaborated to include either the criterion the decision maker sees as being the proper one or to add realistic detail that relaxes the unrealistic assumption brought into question. The model analysis then needs to be repeated. Now there is a bigger parameter space to explore, but the prior exploration has helped define where to begin in that exploration. If the analysis of the more elaborate model demonstrates that the particular elaboration does not make much difference, that particular elaboration may be dropped from the model when some other elaboration is explored. If it makes a meaningful difference, whether or not it changes the decision, it might be included as the next aspect of reality is added to the model.

This process of increasing model realism continues until the decision maker says "Enough, I must act on what I know now." It may proceed to include a jump from SC models to DN models if the unrealistic SC model assumption of instantaneous contacts with no effect of a contact on who might be contacted next is judged to have the potential to be distorting the decision under consideration.

\section{SUMMARY AND IMPLICATIONS}

We have outlined four classes of population models for infection transmission that can be usefully traversed in the pursuit of five modeling goals. These goals and model forms should be just as relevant to analysis of non-linear population dynamics 
in demography as they are to analysis of infection transmission system dynamics. To construct this set of four model classes we constrained the models to continuous time and made all times to events have Erlang distributions. All of reality cannot be captured when making such restrictive assumptions, but making these assumptions expands the freedom of population scientists to elaborate investigations into the social plane of demographic and epidemiologic data analysis. Since time is in fact continuous, and since many time to event distributions are determined by rates of component events, these restrictions are not too confining.

The approach we propose would have population scientists pursuing their science in the following way. They would develop population theory; manifest that theory in models; explore those models to find ways that data can be gathered to test the theory; and then elaborate ever more detailed and realistic theory and models. In other words, we envision a process of interaction between theoretical models and data that characterize the most progressive sciences but is most often not a characteristic of demography and epidemiology.

Software that facilitates transition between model forms and addition of realistic geographic and social spaces to population models should help more population scientists purse this path to new knowledge. Such software will have no effect, however, unless population scientists become more motivated to pursue theory based upon non-linear population processes. Use of IEH or DNW model forms could help provide the needed motivation. With these model forms, one can evaluate the validity of analytic methods that one is using on real world data. In the analysis of data generated by model processes as opposed to real world processes, one knows the true state of affairs leading to the data. Pursuing the validation of real world analytic approaches in this way will let population scientists see how informative or how deceptive their usual analytic methods are. They will be able to see that by making extreme simplifying assumptions such as no interactions between individuals there is much that is lost. That should then motivate the pursuit of more integral analyses that incorporate realistic aspects of population systems.

\section{REFERENCES}

1. KoOpman, J.S. \& I.M. Longini. 1994. Ecological effects of individual exposures and non-linear disease dynamics in populations. Am. J. Public Health 84(5): 836-842.

2. Susser, M. \& E. Susser. 1996. Choosing a future for epidemiology. 2. From black box to Chinese boxes and eco-epidemiology. Am. J. Public Health 86: 674.

3. Koopman, J.S. 1996. Emerging objectives and methods in epidemiology. Am. J. Public Health 86(5): 630-632.

4. Susser, M. 1998. Does risk factor epidemiology put epidemiology at risk? Peering into the future. J. Epidemiol. Commun. Health 52: 608-611.

5. Schwartz, S., E. Susser \& M. Susser. 1999. A future for epidemiology? Annu. Rev. Public Health 20: 15-33.

6. BeCKer, N.G. 1993. Parametric inference for epidemic models. Math. Biosci. 117: 239-251.

7. Britton, T. 1998. Estimation in multitype epidemics. J. Roy. Stat. Soc. Ser. B, Stat. Methodol. 60: 663-679.

8. Andersson, H. \& T. Britton. 2000. Stochastic Epidemic Models and Their Statistical Analysis. Springer-Verlag, New York.

9. Poole, D. \& A.E. RAfTery. 2000. Inference for deterministic simulation models: the Bayesian melding approach. J. Am. Stat. Assoc. 95: 1244-1255. 
10. Anderson, R.M. \& R.M. May. 1992. Infectious Diseases Of Humans: Dynamics And Control. Oxford University Press, Oxford.

11. Garnett, G.P. \& R.M. Anderson. 1996. Sexually transmitted diseases and sexual behavior: insights from mathematical models. J. Infect. Dis. 174(Suppl. 2): S150161.

12. Aral, S.O. \& R. Roegner. 2000. Mathematical modeling as a tool in STD prevention and control. Sex. Transm. Dis. 27: 556-557.

13. Chick, S.E., A.L. Adams \& J.S. Koopman. 2000. Analysis and simulation of a stochastic, discrete-individual model of STD transmission with partnership concurrency. Math. Biosci. 166: 45-68.

14. Kretzschmar, M. \& K. Dietz. 1998. The effect of pair formation and variable infectivity on the spread of an infection without recovery. Math. Biosci. 148(1): 83-113.

15. Kretzschmar, M., J.C. Jager, D.P. Reinking, et al. 1994. The basic reproduction ratio R0 for a sexually transmitted disease in a pair formation model with two types of pairs. Math. Biosci. 124(2): 181-205.

16. Diekmann, O., A.P. Heesterbeek \& J.A.J. Metz. 1990. On the definition and the computation of the basic reproduction ration $\mathrm{R} 0$ in models for infectious diseases in heterogeneous populations. Math. Biol. 28: 365-382.

17. Koopman, J.S., J.A. JACQuez, C.P. Simon, et al. 1997. The role of primary HIV infection in the spread of HIV through populations. J. AIDS Hum. Retroviruses 14: 249258.

18. Rothenberg, R.B. \& J. NARramore. 1996. The relevance of social network concepts to sexually transmitted disease control. Sex. Transm. Dis. 23: 24-29.

19. Rothenberg, R.B., C. Sterk, K.E. Toomey, et al. 1998. Using social network and ethnographic tools to evaluate syphilis transmission. Sex. Transm. Dis. 25: 154-160.

20. Morris, M. \& M. Kretzschmar. 1995. Concurrent partnerships and transmission dynamics in networks. Social Networks 17: 299-318.

21. KretZschmar, M. \& M. Morris. 1996. Measures of concurrency in networks and the spread of infectious disease. Math. Biosci. 133: 165-195.

22. Koopman, J.S., S.E. Chick, C.P. Riolo, et al. 2001. Stochastic effects of disseminating versus local transmission of infection. Math. Biosci. Publication pending.

23. Koopman, J.S., S.E. Chick, C.S. Riolo, et al. 2000. Modeling contact networks and infection transmission geographic and social space using GERMS. Sex. Transm. Dis. 27: $617-625$.

24. DietZ, K. \& K.P. Hadeler. 1988. Epidemiological models for sexually transmitted diseases. J. Math. Biol. 26: 1-25.

25. Schriber, T.J. 1991. An Introduction To Simulation Using GPSS/H. John Wiley and Sons.

26. Benaim, M. \& M.W. Hirsch. 1999. Differential and stochastic epidemic models. Fields Institute Commun. 21: 31-44.

27. Vlad, M.O. \& B. Schonfisch. 1996. Mass action law versus local contagion dynamics. A mean-field statistical approach with application to the theory of epidemics. J. Phy. A. Math. Gen. 29: 4895-4913.

28. ONG, C.S., D.L. EISLER, S.H. GoH, et al. 1999. Molecular epidemiology of cryptosporidiosis outbreaks and transmission in British Columbia, Canada. Am. J. Trop. Med. Hyg. 61(1): 63-69.

29. WIDMER, G. 1998. Genetic heterogeneity and PCR detection of Cryptosporidium parvum. Adv. Parasitol. 40: 223-239.

30. Chick, S.E., J.S. Koopman, S. Soorapanth \& M.E. Brown. 2001. Infection transmission system models for microbial risk assessment. Sci. Total Environ. 274(1-3): 197-207.

31. Frost, F. \& T. Muller. 1999. Two City Cryptosporidium. American Water Works Association Research Foundation, Denver, Colorado.

32. NeE, S., E.C. Holmes, R.M. MAy, et al. 1994. Extinction rates can be estimated from molecular phylogenies. Philos. Trans. Roy. Soc. Lond. B 344: 305-311.

33. Pybus, O.G., A. Rambaut \& P.H. Harvey. 2000. An integrated framework for the inference of viral population history from reconstructed genealogies. Genetics 155: 1429-1437. 